\title{
A PLANE CONTINUUM NO TWO OF WHOSE NON- DEGENERATE SUBCONTINUA ARE HOMEOMORPHIC: AN APPLICATION OF INVERSE LIMITS
}

\author{
R. D. ANDERSON AND GUSTAVE CHOQUET ${ }^{1}$
}

1. Introduction. The primary purpose of this paper is to show that in the plane there exists a continuum no two of whose nondegenerate subcontinua ${ }^{2}$ are homeomorphic. We shall give a rather detailed constructive proof of the existence of such a continuum $M$ which does not separate the plane. We shall suggest modifications of this construction to yield (a) such a continuum $M^{\prime}$ every nondegenerate subcontinuum of which does separate the plane and (b) a one-dimensional continuum no two of whose nondegenerate subcontinua are homeomorphic and which contains no nondegenerate subcontinuum embeddable in the plane. The continuum $M$ to be described has two additional properties: (1) $M$ does not contain uncountably many disjoint nondegenerate continua and (2) each nondegenerate subcontinuum of $M$ admits an atomic mapping ${ }^{3}$ onto a continuum which is the sum of finitely many intervals emanating from the origin. From either (1) or (2) it follows that $M$ is hereditarily decomposable.

We shall describe $M$ as the inverse limit set ${ }^{4}$ of a sequence $\left\{M_{i}\right\}$ of plane continua. The construction of $M$ will be such that it will follow from our Theorem I that $M$ is embeddable in the plane and from the unicoherence of $M$ that $M$ does not separate the plane.

The procedure for obtaining $M$ is suggested by the usual construction of an irreducible mapping $\psi$ of a plane continuum $K$ onto the unit interval in the $x$-direction such that $\psi$ is a projection mapping with the collection of nondegenerate inverses dense in $K$. Thus every inverse under $\psi$ is either a point or an interval. The set $K$ can be ob-

Presented to the Society, December 29, 1955; received by the editors December 27, 1955 and, in revised form, March 3, 1958.

1 This research was supported in part by the National Science Foundation, Grant G1013 and in part by the Air Force Office of Scientific Research AF18(600)-1109.

${ }^{2}$ As used in this paper the term continuum means a compact connected metric point set.

${ }^{3}$ A mapping is a continuous transformation. Let $X$ and $Y$ be compact metric spaces. A mapping $f$ of $X$ onto $Y$ is said to be atomic if (1) $f$ is monotone, i.e. pointinverses are connected, and (2) for every continuum $K \subset X$ for which $f(K)$ is nondegenerate, $K=f^{-1} f(K)$. An atomic mapping will be called an $A$-map. If at most a finite number of the inverses of points under an $A$-map are nondegenerate, the $A$ map will be called an $A^{*}$-map.

- See $\S(2)$ for definition. 
tained by considering the closure of the graph of $y=\sin \pi / x$ and a damped localization of the same phenomenon. The procedure used in this paper is also quite similar to that employed by Whyburn [1] in his example of a plane continuum every nondegenerate subcontinuum of which separates the plane.

2. Inverse limits. If $X$ is a point set and $p$ is a point, by $p(X, p)$ will be meant the Hausdorff distance from $X$ to $p$, i.e. the l.u.b. of the distances from $p$ to the points of $X$.

If $X$ and $Y$ are points or point sets, by $d(X, Y)$ will be meant the distance between $X$ and $Y$.

If $X$ is a point set, $D(X)$ will mean the diameter of $X$.

Let $\left\{M_{i}\right\}$ be a sequence of compact metric point sets and, for each $i$, let $f_{i}$ be a mapping of $M_{i+1}$ onto $M_{i}$. Let $M$ be the set of all sequences $\left\{p_{i}\right\}$ where, for each $i, p_{i} \in M_{i}$ and $f_{i}\left(p_{i+1}\right)=p_{i}$. Let $D$ be the collection of all subsets of $M$ such that $d$ is an element of $D$ if and only if for some $i$ and some open set $d_{i}$ in $M_{i}, d$ is the set of all points of $M$ whose $i$ th coordinate is in $d_{i}$. With $D$ as a subbasis, it follows that $M$ is a compact metric space. $M$ is called the inverse limit (set) ${ }^{5}$ of the sequence $\left\{M_{i}\right\}$ with respect to the sequence $\left\{f_{i}\right\}$.

It is not true that if, for each $i, M_{i}$ is embeddable in a space $S$ then $M$ is embeddable in $S$. For instance, a solenoid is the inverse limit of a sequence of circles and is not embeddable in the plane. A criterion for the embeddability of $M$ in $S$ is given here. Such a criterion, while lengthy to state and easy to verify, can be readily applied to certain complicated constructions of continua, particularly in Euclidean space, and thus may be of value in simplifying the descriptions of some other examples than the present one.

Let $f_{i j}$ denote the mapping $f_{i} \cdot f_{i+1} \cdots f_{j-1}$ of $M_{j}$ onto $M_{i}$ and let $f_{i i}$ be the identity mapping of $M_{i}$. For each $i$, let $\tilde{f}_{i}$ be the mapping of $M$ onto $M_{i}$ induced by $f_{i}, f_{i+1}, \cdots$.

THEOREM I. Let the compact sets $M_{i}$ be subsets of a given compact metric space $S$. We suppose that (1) for every $i$ and every $\delta>0$ there exists a $\delta^{\prime}>0$ such that if $j>i ; p, q \in M_{j}$ and $d\left(f_{i j}(p), f_{i j}(q)\right)>\delta$ then $d(p, q)>\delta^{\prime}$ and (2) for every $\epsilon>0$ there exists a number $k$ such that if $p \in M_{k}$, then $D\left(\cup_{j>k} f_{k j}^{-1}(p)\right)<\epsilon$. Then the inverse limit set $M$ is homeomorphic to the subset $A$ of $S$ defined by $A=\bigcap_{i}\left(\bigcup_{k \geqq i} M_{k}\right)^{* 6}$, in this case the sequential limiting set of the sequence $\left\{M_{i}\right\}$.

5 The definition is usually given for $f_{i}$ a mapping of $M_{i+1}$ into $M_{i}$ but in such a case the $M_{i}$ 's can be cut down so as to admit mappings onto. It is usually not required that the $M_{i}$ be metric and that the indices, $i$, form a sequence; we require such because of the applications we have in view.

- * indicates closure. 
Proof of Theorem I. From condition (2) it follows that each point $p=\left\{p_{i}\right\}$ of $M$ is such that the sequence of points $p_{i}$ in $S$ has a sequential limiting point $p^{\prime}$ in $S$. Define $\phi(p)=p^{\prime}$ and $\phi$ is a transformation of $M$ into $S$. If $q=\left\{q_{i}\right\}$ is a point of $M$, then from condition (1) it follows that $\phi(q) \neq \phi(p)$ unless, for each $i, q_{i}=p_{i}$. Hence $\phi$ is one-toone. But again from (2) and the metricity of $S$, if $U$ is an open set in $S$ containing $\phi(p)=p^{\prime}$, then $U$ contains an open set $V$ containing $p^{\prime}$ such that for some integer $e$, every point $q^{\prime}$ of $V$ for which $\phi^{-1}\left(q^{\prime}\right)=q$ $=\left\{q_{i}\right\} \in M$ is such that for $i>e, q_{i} \in U$. Hence $\phi$ is continuous, and since $M$ is compact, is a homeomorphism. But from metricity and (2) it follows that $\phi(M)$ is dense in the sequential limiting set of $\left\{M_{i}\right\}$ and Theorem I follows.

REMARKS. (1) Given a compact subset $A$ of a compact metric space $S$ where $A$ is homeomorphic to the inverse limit of a sequence of compact sets embeddable in $S$, it is not true, in general, that $A$ can be obtained as the sequential limiting set of sets satisfying the conditions of Theorem I. (Take $S$ as the sum of a circle and a solenoid). If, however, $S$ is a manifold, the answer might be affirmative.

(2) Let $M$ be the inverse limit set of a sequence $\left\{M_{i}\right\}$ of compact metric sets with respect to $\left\{f_{i}\right\}$. Then $M$ is homeomorphic to that subset $A$ of $S=\prod_{i} M_{i}$ consisting of the points $x=\left\{x_{i}\right\}$ where for each $i, x_{i}=f_{i}\left(x_{i+1}\right)$. Let $a=\left\{a_{i}\right\}$ be some fixed point of $A$; and for every $i$, let $A_{i}$ be the set of all points $x=\left\{x_{k}\right\}$ where $x_{k}=a_{k}$ for $k>i$ and $x_{k}=f_{k i+1}\left(x_{i+1}\right)$ for $k \leqq i\left(x_{i} \in M_{i}\right)$. There exists a canonical homeomorphism between $A_{i}$ and $M_{i}$ and hence mappings $f_{i}^{\prime}$ of $A_{i+1}$ onto $A_{i}$. It can be verified easily that those subsets $A_{i}$ of $S$ converge to $A$ in the sense of Theorem I. Thus any inverse limit set can be obtained by the procedure of Theorem I.

We note that it is immediate from the definition of atomic mapping that the product of any finite number of atomic mappings is atomic.

Theorem II. If, for each $i, f_{i}$ is atomic, then, for each $i, \tilde{f}_{i}$ is atomic.

Proof. Let $K$ be a continuum of $M$ such that $\tilde{f}_{i}(K)$ is nondegenerate. For every $j \geqq i$, the atomicity of $f_{i j}$ implies that $\tilde{f}_{j}(K)=f_{i j}^{-1} f_{i j} \tilde{f}_{j}(K)$ or $\tilde{f}_{j}(K)=f_{i j}^{-1} \tilde{f}_{i}(K)$ or again $\tilde{f}_{j}(K)=\tilde{f}_{j}\left[\tilde{f}_{i}^{-1} \tilde{f}_{i}(K)\right]$. If we set $B=\tilde{f}_{i}^{-1} \tilde{f}_{i}(K)$ the last relation becomes $\tilde{f}_{j}(K)=\tilde{f}_{j}(B)$. As this relation is valid for every $j>i$ we have $K=B$ which proves the atomicity of $\tilde{f}_{i}$ as was to be shown.

3. Lemmas and the main theorem. It will be assumed that all constructions of this section are in the plane.

An $n$-od, $n>2$, is a continuum homeomorphic to the sum of $n$ distinct intervals of unit length emanating from the origin. 
Lemma 1. Let $\epsilon$ be any positive number. Let $K_{1}, \cdots, K_{j}$ be continua with $K_{1}$ an arc. For each $i, 1 \leqq i<j-1$, let $g_{i}$ be an $A^{*}$-map of $K_{i+1}$ onto $K_{i}$. Let, furthermore, each nondegenerate $g_{i}^{-1}(x)$ for $x \in K_{i}, 1 \leqq i \leqq j-1$, be an $n$-od for some $n$. Let, for $1 \leqq i \leqq j-1, R_{i}$ denote the set of all points $r$ of $K_{i}$ for which $g_{i}^{-1}(r)$ is nondegenerate.

Then there exist a continuum $K_{j+1}$ and an $A^{*}-m a p g_{j}$ of $K_{j+1}$ onto $K_{j}$ such that

(1) $g_{j}^{-1}(x)$, for $x \in K_{j}$, is a point or an $n$-od,

(2) for $x$ and $x^{\prime}$ different points of $K_{j}$ for which $g_{j}^{-1}(x)$ and $g_{j}^{-1}\left(x^{\prime}\right)$ are nondegenerate, $g_{j}^{-1}(x)$ and $g_{j}^{-1}\left(x^{\prime}\right)$ are not homeomorphic to each other nor is either homeomorphic to any set $g_{i}^{-1}\left(x^{\prime \prime}\right)$ for $x^{\prime \prime} \in K_{i}, 1 \leqq i$ $\leqq j-1$,

(3) for $x \in K_{j}, \rho\left(g_{j}^{-1}(x), x\right)<\epsilon$, and

(4) $D(t)<\epsilon$ for each arc $t$ of $K_{j+1}$ for which $d\left(g_{i} \cdots g_{j}(t), R_{i}\right)>\epsilon$, where $i$ is the least integer for which $g_{i} \cdots g_{j}(t)$ is nondegenerate.

Proof. Let $E$ be the set of those points of $K_{j}$ which are emanation points of $n$-ods in $K_{j}$. Let $B$ be the set of points of $K_{j}$ which are not interior points of arcs in $K_{j}$. Each of $E$ and $B$ is finite. Let $n^{\prime}$ be an upper bound on the orders of the points of $K_{1}, \cdots, K_{j}$ as emanation points of $n$-ods in $K_{1}, \cdots, K_{j}$. Let $C$ be a finite set of points $p_{1}, \cdots$, $p_{r}$ of $K_{j}-(B+E)$ such that $D(t)<\epsilon / 3$ for each arc $t$ of $K_{j}-C$ for which $d\left(g_{i} \cdots g_{j-1}(t), R_{i}\right)>\epsilon$ where $i$ is the least integer for which $g_{i} \cdots g_{j-1}(t)$ is nondegenerate.

It is clear from the hypothesis that such a set $C$ exists. Let $n_{1}, \cdots, n_{r}$ be $r$ distinct integers $>n^{\prime}$. For each $i, 1 \leqq i \leqq r$, let $U\left(p_{i}\right)$ be an open circular region about $p_{i}$ as a center and having a radius less than each of $\epsilon / 6, d(C, D+E)$, and $1 / 2$ the least distance between distinct points of $C$.

Where $S$ denotes the plane, we define $K_{j+1} \cdot\left(S-U_{i} U\left(p_{i}\right)\right)$ to be $K_{j} \cdot\left(S-U_{i} U\left(p_{i}\right)\right)$ and $g_{j}$ over such set to be the identity.

Let $s$ be the maximal open arc in $U\left(p_{i}\right) \cdot K_{j}$ which contains $p_{i}$. Let $s^{\prime}$ be a subset of $U\left(p_{i}\right)$ such that (1) $\bar{s}^{\prime}-s^{\prime}=\bar{s}-s$ and (2) $s^{\prime}$ admits an $A^{*}$-map $\psi$ onto $s$ such that $\psi^{-1}\left(p_{i}\right)$ is an $n_{i}$-od $Z_{i}$ and each other inverse is degenerate. It is clear by straightforward construction that such a set $s^{\prime}$ exists. Intuitively, we may think of an $n_{i}$-od in $U\left(p_{i}\right)$ and from the endpoints of $s$ "wrap" open ended arcs about $Z_{i}$ to obtain $s^{\prime}$.

Let $\eta_{i}$ be a mapping of $\bar{U}\left(p_{i}\right)$ onto itself such that (1) $\eta_{i}$ is the identity on $\bar{U}\left(p_{i}\right)-U\left(p_{i}\right)$, (2) $\eta_{i}\left(Z_{i}\right)=p_{i}$, (3) $\eta_{i}\left(s^{\prime}\right)=s$, and (4) $\eta_{i}$ is a homeomorphism from $\bar{U}\left(p_{i}\right)-Z_{i}$ onto $\bar{U}\left(p_{i}\right)-p_{i}$. That such an $\eta_{i}$ exists follows from the obvious existence of a mapping satisfying conditions (1), (2), and (4) and the procedure of following such a map- 
ping with an appropriate homeomorphism of $\bar{U}\left(p_{i}\right)$ onto itself.

For each $i, 1 \leqq i \leqq r$, we define $K_{j+1}$ in $U\left(p_{i}\right)$ to be $\eta_{i}^{-1}\left(K_{j} \cdot U\left(p_{i}\right)\right)$ and define $g_{j}$ to be $\eta_{i}$ over $K_{j+1} \cdot U\left(p_{i}\right)$. Thus $K_{j+1}$ and $g_{j}$ are defined and by definition satisfy the conditions of the lemma.

Lemma 2. There exist sequences $\left\{M_{i}\right\}$ and $\left\{f_{i}\right\}$ such that

(1) $M_{1}$ is an arc;

(2) for each $i, M_{i}$ is a continuum in the unit circle in the plane;

(3) for each $i, f_{i}$ is an $A^{*}$-map of $M_{i+1}$ onto $M_{i}$;

(4) for any $i$ and any $j$ with $p \in M_{i}, q \in M_{j}$ and $p \neq q$, each of $f_{i}^{-1}(p)$ and $f_{j}^{-1}(q)$ is a point or an n-od and the two are homeomorphic only if each is a point;

(5) for no $i$, does there exist an arc $t$ in $M_{i}$ for which $f_{i j}^{-1}(t)$ is an arc for each $j$;

(6) for any $\epsilon>0$ there exists a number $k$ such that if $p$ is any point of $M_{k}$ then, for $j>k, D\left(\cup f_{k j}^{-1}(p)\right)<\epsilon$; and

(7) for each $i$ and any $\delta>0$ there exists $a \delta^{\prime}>0$ such that if $j>i, p, q$ $\in M_{j}$ and $d\left(f_{i j}(p), f_{i, j}(q)\right)>\delta$ then $d(p, q)>\delta^{\prime}$.

Proof of Lemma 2. Lemma 2 is an almost immediate consequence of Lemma 1. We look at the inductive step, given $M_{i}$ and $f_{i-1}$, and investigate to find an appropriate value for $\epsilon$. Conditions (1) to (4) are either trivial to satisy or follow directly from Lemma 1 except that the boundedness condition of (2) can be considered to be implied by what follows. Let $\epsilon<1 / 2^{i}$ and condition (3) of Lemma 1 implies our condition (6). Let $\nu>0$ be a lower bound of the set of distances between points $p$ and $q$ of $M_{i}$ where, for some $r<i$,

$$
d\left(f_{r i}(p), f_{r i}(q)\right)>1 / 2^{i} .
$$

That such a $\nu$ exists follows from the compactness of $M_{j}, j<i$, and the finiteness of the number of mappings concerned. Let $\epsilon<\nu / 2^{i+1}$; then condition (3) of Lemma 1 implies our condition (7). An $\epsilon$ satisfying these two conditions is such that our condition (5) is also satisfied. This follows principally from condition (4) of Lemma 1 and condition (7) as already established. If there were a sequence of arcs violating condition (5), then by (7) the sequence could not be a null sequence and by (4) of Lemma 1 it could not fail to be a null sequence.

Thus Lemma 2 is established.

Theorem III. There exists a plane continuum $M$ such that (1) no subcontinuum of $M$ separates the plane, (2) no two (distinct) nondegenerate subcontinua of $M$ are homeomorphic, and (3) $M$ does not contain uncountably many disjoint nondegenerate subcontinua. 
Proof. The inverse limit set of the sequence $\left\{M_{i}\right\}$ with respect to $\left\{f_{i}\right\}$ of Lemma 2 is such a continuum $M$. That it can be embedded in the plane follows explicitly from Theorem I or a straightforward argument using conditions (6) and (7).

We prove that $M$ satisfies condition (2). Suppose the contrary. Let $X_{1}$ and $X_{2}$ be distinct nondegenerate subcontinua of $M$ and without essential loss of generality assume that $X_{1}$ and $X_{2}$ are disjoint. (For if not, one must contain a subcontinuum $Y_{1}$ not intersecting the other and $Y_{1}$ must be homeomorphic to a subcontinuum $Y_{2}$ of the other.) From Theorem II (or a straightforward argument) it follows that for each $i$ and each $j, j>i$, the maps $f_{i j}$ and $\tilde{f}_{i}$ (of $M$ onto $M_{i}$ ) are $A$-maps. By definition the property of being an $A$-map is hereditary on subcontinua. Let $i_{j}, j=1,2$, be the least number such that $\tilde{f}_{i j}\left(X_{j}\right)$ is nondegenerate. Then each of $\tilde{f}_{i_{1}}\left(X_{1}\right)=S_{i}$ and $\tilde{f}_{i_{2}}\left(X_{2}\right)=S_{2}$ is an $n$-od or an arc. $S_{1}$ and $S_{2}$ do not intersect but they may be homeomorphic. For $j=1,2$, let $i_{j}^{\prime}$ be the least number greater than $i_{j}$ for which $\tilde{f}_{i^{\prime}}\left(X_{j}\right)$ is not homeomorphic to $\tilde{f}_{i^{\prime}-1}\left(X_{j}\right)$ (and by condition (5) of Lemma 2 such a number must exist). Let $S_{j}^{\prime}$ be $\tilde{f}_{i^{\prime}}\left(X_{j}\right), j=1,2$. Then $S_{1}^{\prime}$ contains an $n$-od $T$ which is an inverse set of the $A^{*}$-map $f_{i_{1} i_{1}{ }^{\prime}}$ of $S_{1}^{\prime}$ onto $S_{1}$ and $S_{2}^{\prime}$ contains no similar $n$-od as an inverse set of the $A^{*}$-map $f_{i_{2} i_{2}}$ of $S_{2}^{\prime}$ onto $S_{2}$.

But we note that an $A$-map of $X_{1}$ or $X_{2}$ onto an arc or $n$-od induces a unique (atomic) decomposition of $X_{1}$ or $X_{2}$ (except with respect to order). Thus if $X_{1}$ and $X_{2}$ are homeomorphic, $S_{1}$ and $S_{2}$ must be homeomorphic and under some such induced homeomorphism of $S_{1}$ onto $S_{2}$ the inverses of corresponding points of $S_{1}$ and $S_{2}$ under $f_{i_{1} i_{1}{ }^{\prime}}$ and $f_{i_{2} i_{2}{ }^{\prime}}$ respectively must be homeomorphic. But such is impossible and hence $X_{1}$ and $X_{2}$ are not homeomorphic.

The fact that no subcontinuum $X_{1}$ of $M$ separates the plane follows ${ }^{7}$ from the hereditary decomposability and hereditary unicoherence of $M$ (or $X_{1}$ ) which follow immediately from the above considerations.

Property (3) follows easily. If $Z$ were an uncountable collection of disjoint nondegenerate subcontinua of $M$, then for some uncountable subcollection $W$ of $Z$ there must exist an integer $i$ such that for every $w \in W, f_{i}(w)$ is nondegenerate. Hence $f_{i}(M)=M_{i}$ must contain uncountably many disjoint nondegenerate continua, an impossibility. Theorem III is proved.

${ }^{7}$ For suppose a continuum $X$ separates points $p$ and $q$ in the plane. Then some subcontinuum $X^{\prime}$ of $X$ is irreducible with respect to separating $p$ from $q$. But $X^{\prime}$ is decomposable and hence is the sum of its proper subcontinua $Y_{1}$ and $Y_{2}$. Neither of these separates $p$ from $q$ so $Y_{1} \cap Y_{2}$ is not connected and $X^{\prime}$ is not unicoherent. 
4. Two further theorems. We now state Theorems IV and V and suggest how the proof of Theorem III can be modified to yield them.

Theorem IV. There exists a plane continuum $M^{\prime}$ such that (1) every nondegenerate subcontinuum of $M$ separates the plane and (2) no two distinct nondegenerate subcontinua of $M$ are homeomorphic.

The condition (3) of Theorem III follows from condition (1) of this theorem.

In the proof of Theorem IV, in lieu of an $n$-od we would use a continuum which is the sum of finitely many simple closed curves all and each pair having exactly one point in common. Call such a continuum a loop-set. $M_{1}^{\prime}$ could be taken to be a simple closed curve. The rest of the ideas of the proof go through noting that $S_{1}$ and $S_{2}$ of the proof of Theorem III would become subcontinua of a loop-set.

THEOREM V. There exists a one-dimensional continuum $M^{\prime \prime}$ such that (1) no nondegenerate subcontinuum of $M^{\prime \prime}$ is embeddable in the plane (2) no two distinct nondegenerate subcontinua of $M^{\prime \prime}$ are homeomorphic and (3) $M$ does not contain uncountably many disjoint nondegenerate subcontinua.

In proving Theorem $\mathrm{V}$ we would introduce a set which is an $n$-od plus a 1 -skeleton of a 4 -simplex, the two sets having just the branch point of the $n$-od in common with a vertex of the 1 -skeleton of the 4-simplex. Then every nondegenerate subcontinuum would contain a continuum admitting an atomic map onto a 1-skeleton of a 4simplex and thus as can be shown, would not be embeddable in the plane.

\section{Reference}

1. G. T. Whyburn, A continuum every subcontinuum of which separates the plane, Amer. J. Math. vol. 52 (1930) pp. 319-330.

The University of Pennsylvania,

The University of Paris AND

The InStitute for Advanced Study 